\title{
Identification of Lane-Change Maneuvers in Real-World Drivings with Hidden Markov Model and Dynamic Time Warping
}

\author{
Lars Klitzke*, Carsten Koch ${ }^{\dagger}$ and Frank Köster*
}

\begin{abstract}
For the introduction of new automated driving functions, the systems need to be verified extensively. A scenario-driven approach has become an accepted method for this task. But to verify the functionality of an automated vehicle in the simulation in a certain scenario such as a lane change, characteristics of scenarios need to be identified. This, however, requires to extract lane-change from real-world drivings accurately. For that purpose, this work proposes a novel framework for lane-change identification by combining multiple unsupervised learning methods. To represent various types of lane changes, the maneuver is split up into primitive driving actions with an Hidden Markov Model (HMM) and Divisive Hierarchical Clustering (DHC). Based on them, lane change maneuvers are identifier using Dynamic Time Warping (DTW). The presented framework is evaluated with a realworld test drive and compared to other baseline methods. With a $F_{1}$ score of $98.01 \%$ in lane-change identification, the presented approach outperforms the other approaches.
\end{abstract}

Index Terms-Lane-change Maneuver, Hidden Markov Model, Dynamic Time Warping, Divisive Hierarchical Clustering, Automated Driving

\section{INTRODUCTION}

In the last decades, the domain of autonomous driving has gained much attention from the research community. The primary focus was on developing and improving automated driving functions, whereas the topic of testing these systems was only a niche. In recent years, however, that changes drastically, and more effort was put into developing new validation methodologies [1]. This was due to the tedious testing process, and the enormous overall effort to verify the system's functionality. Indeed, the research focus on testing methods shifted to scenario-based validation approaches.

For a scenario-based validation methodology, however, a set of scenarios is essential [2]. Although several studies attempt to identify scenarios [3], [4], [5], [6], [7] and describing them appropriately [8], this topic is still an open issue and demands further investigation.

A typical scenario or maneuver on motorways are lanechanges. The analysis of lane-changes is broadly discussed in the academic domain for several decades. Vehicles performing lane-changes can affect the overall traffic flow [9] and are one of the main reasons for accidents on highways [10]. Thus, they pose a severe risk to traffic participants.

With an increasing level of automation, the Automated Vehicle (AV) has to conduct lane-changes safely-even under uncertainty. Due to this, studies focused on analyzing

\footnotetext{
* Lars Klitzke and Frank Köster are with the German Aerospace Center (DLR), Institute of Transportation Systems, Braunschweig, Germany \{lars.klitzke, frank.koester\}@dlr.de.

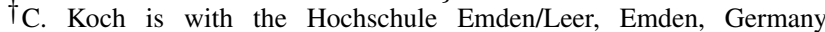
carsten.koch@hs-emden-leer. de.
}

lane-change maneuvers to derive driving models that can be integrated into the AV for trajectory planning [10] or to assess the AV's performance and safety [11]. For both research fields, however, the identification of lane-changes in large-scale databases from naturalistic field operational tests is a prerequisite.

Several works employ supervised learning methods for the identification of lane-changes. In recent years, the Support Vector Machine (SVM) [12], [13], [14] and Artificial Neuronal Network (ANN) [15] were utilized. For lane-change classification, however, the data need to be prepared with a sliding window approach, which size is another hyperparameter to tune. Another drawback of using supervised learning methods are the required ground truth information for training. To overcome this, unsupervised learning methods were employed recently. Kruber et al. used random forest combined with hierarchical clustering to identify scenarios in simulation data [6]. Probabilistic-based methods such as the HMM were also proposed for, e.g., driving style analysis [16]. Nevertheless, the final cluster interpretation has to either be performed manually by an expert [6] or compared to predefined characteristics [16] since the semantic meaning is lost after clustering.

\section{A. Contribution}

This work presents a novel framework for the identification of lane-change maneuvers. Instead of using supervised learning methods to extract lane-change maneuvers, in this work, test-drives are clustered with an HMM into more trivial driving actions, the driving primitives $(D P)$ and pattern matching is employed for the identification.

Since a significant drawback in clustering with nondeterministic methods such as the HMM is the different labeling, we propose a method to recover the semantics of driving primitives. Moreover, to model lane-change maneuvers based on the DPs, this work proposes a patternrecognition based approach. For each maneuver to identify, specific patterns are created. Furthermore, the time-series is clustered according to the DPs using Divisive Hierarchical Clustering (DHC). DTW is then employed to find the most likely maneuver based on the defined patterns.

\section{B. Paper Structure}

In Section II, the proposed framework for lane-change identification based on DPs is elaborated.Afterward, the framework is evaluated in Section III. Finally, the work concludes with a summary and an outlook on further works in Section IV. 


\section{LANE-CHANGE IDENTIFICATION FRAMEWORK}

The proposed approach for lane-change identification is a multi-level framework that consists of multiple unsupervised learning methods for clustering (HMM, DHC and $k$-means) into DPs and DTW for the final identification (see Fig. 2).

The stages are derived from a typical lane-change maneuver exemplarily depicted in Fig. 1. In the first state Idle, the vehicle drives in the center of the lane and transitions into the state Approach if it approaches either the left or right lane marking. If any vehicle side crosses the street marking, the vehicle is in the state Cross until the vehicle's center crosses that marking and changes to the Change state. Hence, the vehicle is changing the lane if the majority of the vehicle is on the new lane. The maneuver finishes if the second side of the vehicle crosses that marking, which is indicated by the Depart state, followed by the Settle state denoting the vehicle driving in the middle of the new lane. From a lane-
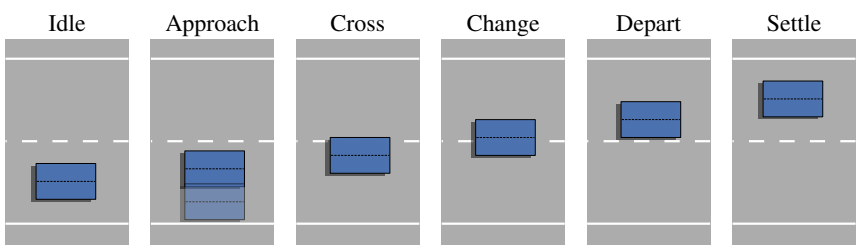

Fig. 1: A simple lane-change maneuver consists of six stages. They are used for maneuver sequence partitioning and classification.

oriented point of view, a typical lane-change maneuver does not consist of six but four states. The states Idle and Settle, as well as Approach and Depart, are more or less the same since the absolute relative position of the vehicle in the lane equal. Hence, this work uses the following four states.

- Idle: The vehicle drives in the center of the lane

- Approach: The vehicle approaches the left or right lane marking

- Cross: The left or right side of the vehicle crosses the right or left lane marking.

- Change: The vehicle side crosses the lane marking, which was previously crossed by the other vehicle side.

Based on this concept, a multi-level framework is proposed to perform maneuver classification (see Fig. 2). In the following, each level is described briefly.

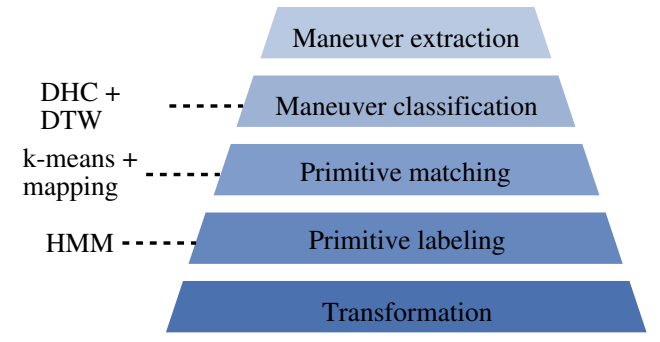

Fig. 2: The proposed framework for lane-change identification based on driving primitives.

\section{A. Transformation}

To divide the lane-change maneuver into the driving primitives, features need to be selected that appropriately represent the vehicle's state. In this work, the distances from the vehicle's center to the left $d_{c l}$ and right $d_{c r}$ lane marking are employed that are provided by the vast majority of frontfacing cameras. However, instead of using these distances, features are derived that are independent of the vehicle and lane width. That allows the presented approach to function on roads with arbitrary lane widths and for different vehicles.

The first feature is the normalized distance from the vehicle center to the lane center $d_{c}$ (see Fig. 3). This feature represents the lateral displacement of the vehicle in the lane. Based on the distance between the vehicle's center and the left lane marking, the normalized lane-center distance $d_{c}$ is defined as

$$
d_{c}=\frac{\frac{w_{l}}{2}-d_{c l}}{\frac{w_{l}}{2}}=1-\frac{2 d_{c l}}{w_{l}}, d_{c} \in \mathbb{Q}
$$

with $d_{c l}$ as the distance to the left marking and $w_{l}$ the lane width. The latter is estimated using the distances provided by the on-board system $\left(d_{c l}\right.$ and $\left.d_{c r}\right)$. The lane width

$$
w_{l}=\left|d_{c r}-d_{c l}\right|
$$

is the absolute sum of the distances $d_{c l} \in \mathbb{R}$ and $d_{c r} \in \mathbb{R}$ to the left and right lane markings since $d_{c r}<0$ and $d_{c l}>0$.

Although the normalized distance $d_{c}$ may be used for partitioning only, we propose to use another feature $m$ to divide the four states further onto two clusters. Due to this, the HMM will more likely be able to represent the driving primitives. The first cluster represents the vehicle moving within a lane (states Idle and Approach), whereas in the second one, the vehicle moves between two lanes (Cross and Change). For that purpose, the normalized distances $d_{l}$ and $d_{r}$ (see Fig. 3) are used defined as

$$
d_{l}=\frac{\left(d_{c l}-\frac{w_{v}}{2}\right)}{w_{l}} \quad d_{r}=\frac{\left(d_{c r}+\frac{w_{v}}{2}\right)}{w_{l}} .
$$

since the maximum value of $d_{l}$ and $d_{r}$ is $w_{l}-w_{v} / 2$. That is because if the center of the vehicle crosses a lane marking, the on-board system changes the left and right lane markings to represent the new lane boundaries. In Fig. 1, for instance, the left marking is the dashed one and the right marking the solid line until the vehicle transitions in the Change state. Afterward, the left lane is the top solid line and the right lane marking the dashed one.

The distances from the vehicle sides to the lane markings $\left(d_{l}\right.$ and $d_{r}$ ) are employed to mark the vehicle's current substate with the feature $m \in\{-1,0,1\}$ where

$$
m= \begin{cases}-1 & d_{l}<0 \\ 1 & d_{r}>0 \\ 0 & \text { else }\end{cases}
$$




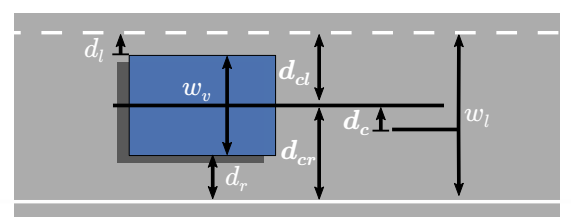

Fig. 3: The features used for the identification of lanechange maneuvers are derived from the distances between the vehicle center and the left $d_{c l}$ and right $d_{c r}$ lane markings.

\section{B. Primitive labeling}

To partition the lane-change maneuver into sequences, each of which represent one of the four states of the vehicle defined previously, this work employs an HMM.

A HMM is typically used if the system's internal state is not accessible, but the system emits information. This information can be used to derive the system's internal state under the condition that the number of states is known. In this work, the system is the vehicle, the internal states are the driving primitives, and the system emits information about the distance to the next lane markings. By defining the number of internal states of the HMM, the parameters of the HMM are derived using an EM algorithm and a driving sequence. The result of an exemplary drive is shown in the left image of Fig. 4 representing the vehicle's lateral displacement distribution for each driving primitive (DP) within the drive generated derived using the Viterbi algorithm.

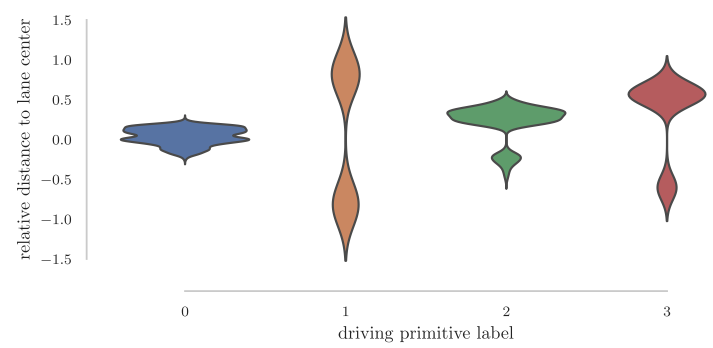

Fig. 4: The result of the HMM with a sequence partitioned into four driving primitives.

\section{Primitive matching}

The next step is to partition the driving sequence into driving primitives. Since the result of the HMM may be different if the parameters are estimated multiple times, the correct labels are derived from the driving sequence.

Let $P=\left\{p_{1}, p_{2}, p_{3}, p_{4}\right\}$ represent the driving primitives with $p_{i}=x_{1}, x_{2}, \ldots, x_{n}$ as the normalized distances for each driving primitive. Let $L=\{0,1, \ldots, n\}$ denote the labels for the primitives and $M_{L}=\{(l, p) \mid l \in L, p \in P\}$ a set of tuples and each tuple consists of a label $l$ and a driving primitive $p$. Furthermore, let $M_{L}(p) \rightarrow l$ be the label $l \in L$ associated with the primitive $p$. We have to determine the correct labels $\bar{L}$ for each primitive. For that purpose, we estimate the absolute mean relative distances from the vehicle center to the lane center $\widetilde{m}=\left\{\widetilde{m}_{1}, \ldots, \widetilde{m}_{4}\right\}$ with

$$
\widetilde{m}_{i}=\frac{1}{\left|p_{i}\right|} \sum_{x}^{p_{i}}|x| .
$$

The correct driving primitives labels $\bar{L}=\left\{l_{1}, \ldots, l_{4}\right\}$ are the indices of the sorted set of means

$$
\bar{L}=\arg \operatorname{sort} \widetilde{m}
$$

with arg sort giving the location of the centers in $\widetilde{m}$ to sort the set in ascending order. The set of tuples $M_{\bar{L}}$ now defines the correct primitive label.

The next step is to determine the direction of the driving primitive since we currently only know the type. Despite the Idle primitive, all primitives follow a bimodal distribution with each cluster representing the direction of that primitive (see Fig. 4). Since the clusters do not overlap, $k$-means is employed to cluster each driving primitive $p_{i} \in P, i>0$ despite the first one, into two clusters $c_{i}=\left\{c_{i}^{1}, c_{i}^{2}\right\}$ with $c_{i}^{j}, j \in\{1,2\}$ denoting the cluster mean and $c_{i}^{1}>c_{i}^{2}$. The primitive direction of a sample $x$ is encoded in the sign of the label $l$ of the primitive $p_{x}$ with

$$
l\left(x, p_{x}\right)=\left\{\begin{array}{l}
M_{\bar{L}}\left(p_{x}\right) \text { if }\left|x-c_{1}\right|<\left|x-c_{2}\right| \\
-M_{\bar{L}}\left(p_{x}\right) \text { else }
\end{array}\right.
$$

according to the distance of the sample to the primitive's cluster means. The result of the label matching and primitive direction estimation is depicted in Fig. 5 with the primitives sorted by their mean distance and the primitive colors denoting the direction.

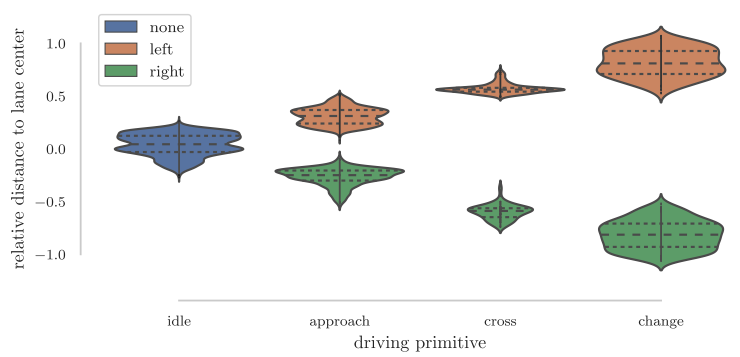

Fig. 5: The partitioned driving sequence with the correctly matched primitives and direction information.

\section{Maneuver classification}

The next step in the framework is to find lane-changes based on the driving primitives. Remark that each driving primitive is represented with a label $l$ and the sign of the label denotes the direction. Hence, we have a time-series of labels we want to classify as lane-changes, i.e., we want to identify the pattern of a lane-change.

For pattern matching in time-series, Dynamic Time Warping (DTW) and more specifically, the nearest neighbor algorithm with DTW, is, although already proposed in the 70s [17], still the benchmarking algorithms for time series classification. It allows to compare signals with different lengths and provides a distance metric for comparison. 
For that purpose, however, Divisive Hierarchical Clustering (DHC) is first employed to divide the time series into smaller chunks based on the driving primitive's label. In this work, the fastDTW implementation [18] for DTW is used, which speeds up the process.

At first, let $H=\left\{h_{1}, \ldots, h_{k}\right\}$ be a set of patterns to match with the time-series $X=x_{1}, x_{2}, \ldots, x_{n}$. The pattern $h_{l}=$ $\{1,2,3,-3,-2,-1\}$ represent a lane-change to the left and $h_{r}=\{-1,-2,-3,3,2,1\}$ to the right with , describing the series of driving primitives in the maneuver. We can employ DTW to map both patterns on the time-series. Let $\mathrm{dtw}(\mathrm{h}, \mathrm{X})$ return the distance mapping the pattern $h$ on the time-series $X$. We search for the pattern with the smallest distance.

$$
\underset{h \in H}{\arg \min } \operatorname{dtw}(h, X)
$$

An example is depicted in Fig. 6 showing a lane-change to the left with the normalized distance to the lane center (in blue) and the driving primitive (orange). The matrix with the accumulated cost to map the value $h_{i}$ of the pattern $h$ at index $i$ to the value $x_{j}$ at the $j$ index in the timeseries is shown on the right with $x$ as the time-series of driving primitive labels. The top image represents the matrix for the left lane-change pattern $h_{l}$ and the bottom for the right $h_{r}$ with the path of lowest cost represented in red. It is evident that the path for the left lane-change pattern has a smaller cost than the one for the right pattern (2.0 vs 63.0 ), and thus, the time-series is classified as left lane-change. The proposed approach for maneuver identification has the
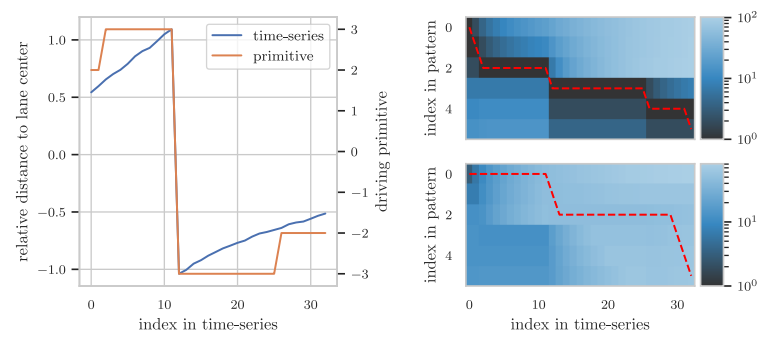

Fig. 6: Lane change classification using Dynamic Time Warping. Left: The time series of the lane-change and the driving primitives. Right: The accumulated cost matrix for mapping the left (top) and the right lane-change (bottom) with the chosen path (in red).

benefit that further maneuvers of interest can be identified by merely adding further patterns to the list $H$.

\section{E. Maneuver extraction}

The intervals generated by the previous steps may not represent the real lane-change accurately. A typical case is if the vehicle is in the state Approach for a lengthy period before the actual lane-change. Thus, a post-processing step is required for maneuver interval extraction. The proposed maneuver extraction approach is elaborated in the following for the lane-change maneuver depicted in Fig. 7 with the blue line as the normalized distance from the vehicle to the lane center. The approach aims at extracting the interval

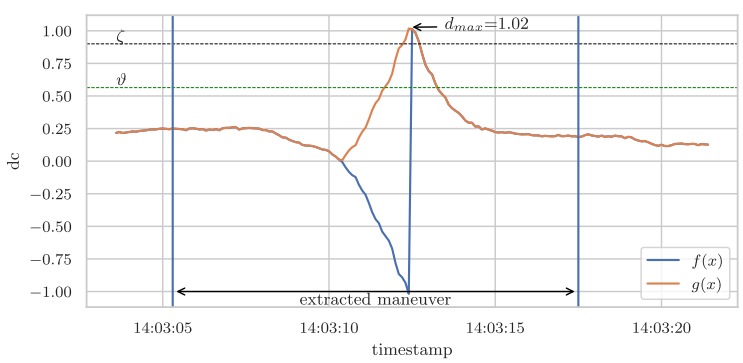

Fig. 7: The absolute normalized distance is used to fine-tune the extracted maneuver interval.

$\left(t_{s}, t_{e}\right)$ represented with the two vertical lines in Fig. 7. Let $f(t)=d_{c}(t)$ be the normalized distance from the vehicle center to the lane center at time $t$ in a time window $w=\{t-\Delta t, \ldots, t+\Delta t\}$ with a duration of $2 \Delta t$ so that $t \in w$. Since the aim is to fine-tune the point in time of the maneuver start and end, the window is equally and generously enlarged in both directions by $\widetilde{\Delta t}=8$ seconds. Then, let $g(t)=|f(t)|$ represent the absolute normalized distance denoted as the orange line in Fig. 7. The first step is to split up the signal into two parts representing the time before and after the lane crossing.

For that purpose, the time and value $t_{\max }, d_{\max }$ of the signal peak is estimated in the window denoting the point in time of the lane crossing. Afterwards, the signal window is split up into the left $w_{l}=\left\{t_{1}, \ldots, t_{l}\right\}$ and right $w_{r}=$ $\left\{t_{r}, \ldots, t_{|w|}\right\}$ sub-window. Since the vehicle may drive for a longer period between the two lanes, the start of the windows $\left(t_{l}, t_{r}\right)$ is determined by

$$
t_{l}=\max \left(\left\{t \mid t \in w, t<t_{\max } \wedge g(t)<d_{\max }\right\}\right)
$$

and

$$
t_{r}=\min \left(\left\{t \mid t \in w, t>t_{\max } \wedge g(t)<d_{\max }\right\}\right)
$$

that is the earliest and latest time where the signal is smaller than the threshold $\zeta$ (see the upper horizontal line in Fig. 7).

The next step is to find the situation where the vehicle's heading recovers to the lane's heading. For that purpose, the windows are stripped down to $w_{l}=\left\{t_{0}, \ldots, t_{l}-t_{o, l}\right\}$ and $w_{r}=\left\{t_{r}+t_{o, r}, \ldots, t_{|w|}\right\}$ with $t_{o, l}, t_{o, r}$ as the offsets in both sub-windows likewise determined by (9) and (10) with the threshold $\vartheta$ (see Fig. 7), since we assume that the sequence contains a lane-change maneuver and that lanechanges will always end after the vehicle crosses the lane marking. Due to this, $\vartheta$ is the mean of the cluster in the HMM represented by the state Crossing. In order to find the start and end of the maneuver, each sub-window is partitioned into $m=\left\lfloor\frac{|w|}{k}\right\rfloor$ bins $b_{1}, b_{2}, \ldots, b_{m}$ with $|w|$ as the size of the sub-window $w$ and $k=\max \left(\left[3, t_{o}\right]\right)$ as at least three or the previously determined offset. The latter ensures, that partitions have a valid size for the follow up steps. Also note that before partitioning, a convolution is applied to the signal with a kernel of size 7 for smoothing. Furthermore, the signal is normalized, so that the first value 


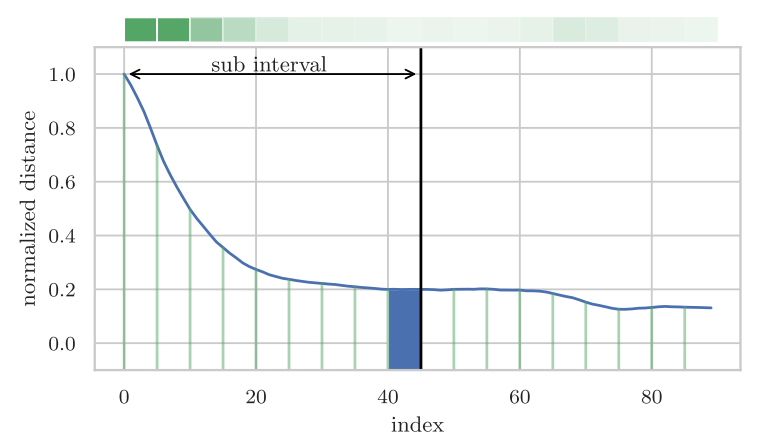

Fig. 8: The end of the maneuver is determined by the signal partition with the lowest average signal change (blue box).

is one. For each bin $b_{i}, i=1, \ldots, m$, of the sub-window $w$ the average signal derivation is determined by

$$
b_{i}=\frac{1}{k} \sum_{j=i k+1}^{(i+1) k} f^{\prime}\left(w^{j}\right)
$$

with $f^{\prime}(t)$ as the derivation of $f(t)$ and $w^{j}$ as the $j t h$ time in the sub-window $w$ since we are interested in the partition with the lowest average signal change. The process is illustrated exemplarily for the right sub-window $w_{r}$ in Fig. 8 with the green vertical lines denoting the partition borders. The mean signal change for each partition is illustrated with the color-coded boxes at the top of the figure. The brighter the box, the lower the average signal derivation.

The last step is to find the $i t h$ bin with the lowest average signal change in both windows $i_{l, r}$

$$
i_{l}=\underset{j \in\left\{1, \ldots, k_{l}\right\}}{\arg \min } b_{l, j} \quad i_{r}=\underset{j \in\left\{1, \ldots, k_{r}\right\}}{\arg \min } b_{r, j}
$$

to finally estimate the maneuver interval $\left(t_{s}, t_{e}\right)$ by

$$
\begin{aligned}
& t_{s}=t_{\text {max }}-t_{l}-t_{o, l}-\left(i_{l}+1\right) k_{l} \\
& t_{e}=t_{\text {max }}+t_{r}+t_{o, r}+\left(i_{r}+1\right) k_{r}
\end{aligned}
$$

depicted as the horizontal arrow in Fig. 7.

\section{Evaluation}

For the evaluation of the proposed lane-change identification framework, a test drive on a motorway with a duration of approximately 2.5 hours was chosen. The data was collected as part of the research project FASva [19]. During the drive, 205 lane-changes took place with 101 lane-changes to the left and 104 to the right. The lane-changes were manually labeled by inspecting the images of the vehicle's front camera. The start of a lane-change is therefore defined as the point in time where the vehicle's heading is changing towards the target lane and, in case of multiple consecutive lane-changes, the vehicle position is near the lane center. The maneuver finishes if the vehicle is on the target lane and the vehicle's heading is recovered to that lane or, in case of multiple consecutive lane-changes, it reaches the center of the lane.

Since the SVM and ANN are widely used in the literature for lane-change identification, they are selected for comparison. To also respect the temporal dependencies in the classification, the sequence $X$ is partitioned into $n$ windows $w_{0}, \ldots, w_{n}$ according to the window size $\Delta_{w}$ so that $n=$ $|X|-\Delta_{w}-1$, i.e., without padding the signal. Thus, for each value $x_{i} \in X$ there is a corresponding window $w_{i}$ which is the feature vector. The predicted label $l_{i} \in\{-1,0,1\}$ is either $b_{i}=1$ for a left, $b_{i}=-1$ for a right or $b_{i}=0$ for no lane-change. Since the performance of both approaches is related to the proper selection of parameters, grid-search is employed to find the best parameter combination. The selected parameters are depicted in Table I.

TABLE I: The model parameters found using grid-search for the SVM and ANN with a subset of the evaluation dataset.

\begin{tabular}{ll|ll}
\hline \multicolumn{2}{r}{ SVM } & \multicolumn{2}{c}{ ANN } \\
\hline Window size & 39 & Window size & 35 \\
Kernel & rbf & Hidden Layers & 3 \\
Gamma & 0.001 & Neurons per Layer & 10 \\
C & 100 & Activation function & tanh \\
- & - & Alpha & 1.0 \\
\hline
\end{tabular}

The maneuver intervals are extracted based on series of equal labels with the first label denoting the start and last one the end time.

\section{A. Metrics}

To qualitatively evaluate the performance of the presented approach, the lane-change classification is treated as a binary classification problem. For that purpose, the approach used in a previous work [20] is adopted to state whether a lanechange was correctly classified or not. Let $\mathbf{t}_{c, i}=\left[t_{c}^{s}, t_{c}^{e}\right]$ denote the ith interval represented as start and end time of the lane-change identified by a classifier $c$ with $c \in$ $\{$ ANN, SVM, HMM+DTW, HMM+DTW-Ex $\}$ and $\mathbf{t}_{g}=\left[t_{g}^{s}, t_{g}^{e}\right]$ the manually identified start and end times. HMM+DTW represent the intervals using only the primitive labels and HMM+DTW-Ex the extended interval extraction method from Section II-E.

A maneuver $\mathbf{t}_{c, i}$ is correctly identified, a true positive $(t \mathrm{p})$, if both interval overlaps and the difference between the ground truth and extracted interval is smaller than a threshold $\Delta t$. If the time difference is, however, greater than $\Delta t$ or do not overlap, the maneuver is a false positive (fp). Furthermore, all maneuvers that were not found by a classifier are false negatives ( $\mathrm{f} n$ ). Based on these metrics, the precision and recall are estimated

$$
\text { precision }=\frac{t \mathrm{p}}{t \mathrm{p}+\mathrm{fp}} \quad \text { recall }=\frac{t \mathrm{p}}{\mathrm{tp}+\mathrm{fn}}
$$

for each classifier and combined using the harmonic mean $\left(F_{1}\right.$ score) given with

$$
F_{1}=2 \cdot \frac{\text { precision } \cdot \text { recall }}{\text { precision }+ \text { recall }}
$$

for the final performance evaluation. 


\section{B. Results}

Since the interval deviation affects the classification precision, the performance is evaluated w.r.t to it. In Fig. 9, the $F_{1}$ score for each classifier and different deviations is shown. Contrary to our expectations, the figure shows that the MLP and SVM lack precision compared with the proposed HMM-based approaches for all $\Delta t$ with a maximum $F_{1}$ score of $38.66 \%$ and $37.35 \%$, respectively. The proposed interval extraction method $(\mathrm{HMM}+\mathrm{DTW}-\mathrm{Ex})$ is superior to the HMM+DTW method, although both approaches tend to the same maximum $F_{1}$ score of $98.01 \%$. Concluding, the results show that the proposed framework is able to identify lanechange with high accuracy. A more detailed analysis of the results is part of future work.

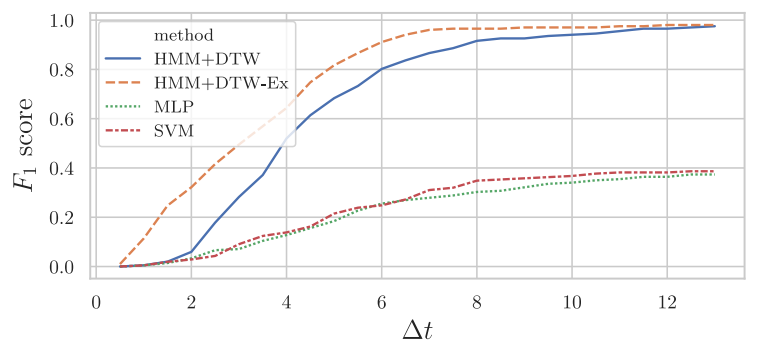

Fig. 9: The classification performance of the proposed approach compared with two state-of-the-art approaches for different maximum maneuver interval deviations.

\section{CONCLUSION}

For the validation of automated driving functions, a scenario-driven approach is a widely accepted method. Therefore, solutions are required to find specific scenarios in real-world drivings. Due to the adverse impact of lane changes on the overall traffic, this work proposes a multilevel framework to identify lane changes on motorways providing information for follow-up analysis. Therefore, a drive is partitioned into driving primitives which are the basis for the maneuver identification.

The proposed approach is evaluated using a test drive on a motorway with 204 lane changes, and the results are compared with other baseline methods showing the efficacy and superiority of the proposed framework with an identification $F_{1}$ score of $98.01 \%$.

In follow-up works, the scalability of the approach and adaptation to other information sources are verified. For that purpose, information of the Testfeld Niedersachsen [21] will be employed to find lane changes for multiple and different types of vehicles.

\section{REFERENCES}

[1] H. Winner, K. Lemmer, T. Form, and J. Mazzega, PEGASUS—First Steps for the Safe Introduction of Automated Driving. Switzerland: Springer International Publishing, 2018, ch. Vehicle Systems and Technologies Development, pp. 185-195.

[2] W. Damm, E. Möhlmann, T. Peikenkamp, and A. Rakow, A Formal Semantics for Traffic Sequence Charts. Cham: Springer International Publishing, 2018, pp. 182-205.
[3] C. Sippl, F. Bock, B. Huber, A. Djanatliev, and R. German, "Identifying relevant traffic situations based on human decision making," in 19. Internationales Stuttgarter Symposium, M. Bargende, H.-C. Reuss, A. Wagner, and J. Wiedemann, Eds. Wiesbaden: Springer Fachmedien Wiesbaden, 2019, pp. 1003-1017.

[4] A. Erdogan, B. Ugranli, E. Adali, A. Sentas, E. Mungan, E. Kaplan, and A. Leitner, "Real- world maneuver extraction for autonomous vehicle validation: A comparative study," in 2019 IEEE Intelligent Vehicles Symposium (IV), June 2019, pp. 267-272.

[5] X. Peng, R. Liu, Y. L. Murphey, S. Stent, and Y. Li, "Driving maneuver detection via sequence learning from vehicle signals and video images," in 2018 24th International Conference on Pattern Recognition (ICPR), Aug 2018, pp. 1265-1270.

[6] F. Kruber, J. Wurst, and M. Botsch, "An unsupervised random forest clustering technique for automatic traffic scenario categorization," in 2018 21st International Conference on Intelligent Transportation Systems (ITSC), Nov 2018, pp. 2811-2818.

[7] C. Roesener, F. Fahrenkrog, A. Uhlig, and L. Eckstein, "A scenariobased assessment approach for automated driving by using time series classification of human-driving behaviour," in 2016 IEEE 19th International Conference on Intelligent Transportation Systems (ITSC), Nov 2016, pp. 1360-1365.

[8] G. Bagschik, T. Menzel, and M. Maurer, "Ontology based Scene Creation for the Development of Automated Vehicles," in 2018 IEEE Intelligent Vehicles Symposium (IV), June 2018, pp. 1813-1820.

[9] Yiguang Xuan and B. Coifman, "Lane change maneuver detection from probe vehicle dgps data," in 2006 IEEE Intelligent Transportation Systems Conference, Sep. 2006, pp. 624-629.

[10] H. Zheng, J. Zhou, Q. Shao, and Y. Wang, "Investigation of a longitudinal and lateral lane-changing motion planning model for intelligent vehicles in dynamical driving environments," IEEE Access, vol. 7, pp. 44783-44802, 2019.

[11] D. Zhao, H. Lam, H. Peng, S. Bao, D. J. LeBlanc, K. Nobukawa, and C. S. Pan, "Accelerated evaluation of automated vehicles safety in lane-change scenarios based on importance sampling techniques," IEEE Transactions on Intelligent Transportation Systems, vol. 18, no. 3, pp. 595-607, March 2017.

[12] P. Dokania, M. Perrollaz, S. Lefevre, and C. Laugier, "Learning-based approach for online lane change intention prediction," 062013.

[13] R. Izquierdo, I. Parra, J. Muñoz-Bulnes, D. Fernández-Llorca, and M. A. Sotelo, "Vehicle trajectory and lane change prediction using ann and svm classifiers," in 2017 IEEE 20th International Conference on Intelligent Transportation Systems (ITSC), Oct 2017, pp. 1-6.

[14] S. Ramyar, A. Homaifar, A. Karimoddini, and E. Tunstel, "Identification of anomalies in lane change behavior using one-class svm," in 2016 IEEE International Conference on Systems, Man, and Cybernetics (SMC), Oct 2016, pp. 004 405-004 410.

[15] N. Monot, X. Moreau, A. Benine-Neto, A. Rizzo, and F. Aioun, "Comparison of rule-based and machine learning methods for lane change detection," in 201821 st International Conference on Intelligent Transportation Systems (ITSC), Nov 2018, pp. 198-203.

[16] W. Wang, J. Xi, and D. Zhao, "Driving style analysis using primitive driving patterns with bayesian nonparametric approaches," IEEE Transactions on Intelligent Transportation Systems, vol. 20, no. 8, pp. 2986-2998, Aug 2019.

[17] H. Sakoe and S. Chiba, "Dynamic programming algorithm optimization for spoken word recognition," IEEE Transactions on Acoustics, Speech, and Signal Processing, vol. 26, no. 1, pp. 43-49, February 1978.

[18] S. Salvador and P. Chan, "Toward accurate dynamic time warping in linear time and space," Intelligent Data Analysis, vol. 11, no. 5, pp. 561-580, 2007.

[19] L. Klitzke, C. Koch, A. Haja, and F. Köster, "Real-world test drive vehicle data management system for validation of automated driving systems," in Proceedings of the 5th International Conference on Vehicle Technology and Intelligent Transport Systems (VEHITS), 2019, pp. 171-180.

[20] L. Klitzke, J. Meyer, T. Leune, C. Koch, and F. Köster, "DAGMaR: A DAG-based Robust Road Membership Estimation Framework for Scenario Mining," in The Second International Workshop on Intelligent Transportation and Connected Vehicles Technologies (ITCVT 2019), Granada, Spain, Oct 2019, pp. 358-365.

[21] F. Köster, J. Mazzega, and S. Knake-Langhorst, "Automatisierte und vernetzte systeme effizient erprobt und evaluiert," ATZextra, vol. 23, no. 5, pp. 26-29, 2018. 